\title{
PHASE TRANSITION NEAR A LIQUID-GAS COEXISTENCE EQUILIBRIUM*
}

\author{
XINFU CHEN ${ }^{\dagger}$ AND XIAO-PING WANG ${ }^{\ddagger}$
}

\begin{abstract}
Effects of small perturbations from a liquid-gas coexistence equilibrium (the Maxwell states) is studied for an isothermal (or isentropic) gas-liquid phase transition in a sealed onedimensional finite length tube, by using a van per Waals model with a viscous-capillary regularization. A matched asymptotic expansion is used to derive formally a linear system satisfied by leading order perturbations. The linear system is solved analytically and checked against numerical simulations. Analyses of the linear system suggest the following: (i) A gas-liquid interface approaches its final destination (determined by mass conservation) in general in a oscillatory manner with frequency determined in part by the speeds of sound in gas and liquid; (ii) Kinetic energies of small initial perturbations will in general be dissipated in the phase transition process, and the system approaches steady states; (iii) In some special cases (for example, the time needed for the sound wave to travel in liquid from the interface to the tube boundary is a rational multiple of that in gas), kinetic energies of certain small perturbations will not be dissipated (in the leading order expansion); in fact, there are infinitely many linearly independent time periodic solutions to the linear system.
\end{abstract}

Key words. phase transition, van der Waals fluid, perturbation analysis

AMS subject classifications. $35,65,76$

PII. S0036139999354285

1. Introduction. The van der Waals system

$$
\left\{\begin{array}{l}
u_{t}=v_{x} \\
v_{t}=-\left\{p(u)+\varepsilon^{2} u_{x x}-\varepsilon \kappa v_{x}\right\}_{x}
\end{array}\right.
$$

with nonmonotone stress relation $p$ and viscous-capillary regularization, has been studied by many authors as a model for dynamical gas-liquid phase transitions in compressible fluid undergoing isothermal motion (see, for example, $[6,8,10,14]$ ).

In this paper, we consider a gas-liquid phase transition in a sealed one-dimensional tube of length $2 a$, i.e., the system (1.1) on a bounded domain $(-a, a)$, with boundary conditions

$$
\left\{\begin{array}{l}
u_{x}(-a, t)=u_{x}(a, t)=0, \\
v(-a, t)=v(a, t)=0
\end{array} \quad t>0 .\right.
$$

Here $v=0$ at $x= \pm a$ refers to the zero velocity and $u_{x}=0$ at $x= \pm a$ refers to the no mass flux boundary condition. We are concerned with initial data which are small perturbations of a gas-liquid equilibrium.

For a steady state of (1.1), $v$ is a constant and $u$ satisfies, for some constant $Q$,

$$
\varepsilon^{2} u_{x x}+p(u)=Q
$$

${ }^{*}$ Received by the editors April 2, 1999; accepted for publication (in revised form) February 11, 2000; published electronically August 3, 2000.

http://www.siam.org/journals/siap/61-2/35428.html

$\dagger$ Department of Mathematics, University of Pittsburgh, Pittsburgh, PA 15260 (xfc@math.pitt. edu). The research of this author was supported in part by National Science Foundation grant DMS9705972.

${ }_{\ddagger}^{\ddagger}$ Department of Mathematics, The Hong Kong University of Science and Technology, Clear Water Bay, Kowloon, Hong Kong (mawang@ust.hk). The research of this author was supported in part by the Research Grant Council of Hong Kong under grant HKUST 6165/97P. 
To accommodate the gas-liquid phase transition, we assume that (1.3) has a solution (in $R$ ) connecting a gas phase and a liquid phase. For this purpose, we assume that the van der Waals function $p(\cdot)$ is a $C^{\infty}((0, \infty))$ function satisfying, for some constants $\alpha^{-}, u_{1}, \alpha^{0}, u_{2}$, and $\alpha^{+}$with $\alpha^{-}<u_{1}<\alpha^{0}<u_{2}<\alpha^{+}$,

$$
\begin{gathered}
p^{\prime}(u)<0 \text { for } u \in\left(0, u_{1}\right) \cup\left(u_{2}, \infty\right) \quad \text { and } \quad p^{\prime}(u)>0 \text { for } u \in\left(u_{1}, u_{2}\right), \\
p\left(\alpha^{-}\right)=p\left(\alpha^{0}\right)=p\left(\alpha^{+}\right)=: Q^{*}, \quad \int_{\alpha^{-}}^{\alpha^{+}}\left(p(u)-Q^{*}\right) d u=0 .
\end{gathered}
$$

The states $\left\{u \mid 0<u<u_{1}\right\}$ and $\left\{u \mid u>u_{2}\right\}$ are called the gas phase and liquid phase, respectively. The states $p=\alpha^{ \pm}$, known as the Maxwell states, are the states in which gas and liquid can stay in equilibrium. We denote $c^{ \pm}=\sqrt{-p^{\prime}\left(\alpha^{ \pm}\right)}$, the speed of sound in $\alpha^{ \pm}$phase.

Solutions of (1.3) on the bounded domain $[-a, a]$ are critical points of the CahnHilliard/van der Waals energy

$$
H(u):=\int_{-a}^{a}\left(\frac{\varepsilon^{2}}{2}\left|u_{x}\right|^{2}+P(u)\right) d x, \quad P(u):=\int_{\alpha^{-}}^{u}\left[p\left(\alpha^{-}\right)-p(s)\right] d s
$$

under a mass constraint $\int_{-a}^{a} u(x) d x=m$. In the one-dimensional setting, Carr, Gurtin, and Slemrod [3] have shown that the minimizer $u^{\varepsilon}$ is monotonic. It is also shown in $[16,17]$ that as $\varepsilon \rightarrow 0$, the solution $u^{\varepsilon}$ (in high space dimension) converges to a function $u_{0}$ which takes only the values $\alpha^{-}$and $\alpha^{+}$with interface between $u_{0}=\alpha^{-}$ and $u_{0}=\alpha^{+}$having minimum area.

Work has been done both numerically and analytically on the structures of the limit states (as $\varepsilon \rightarrow 0$ ) in the dynamic setting. Numerical studies for the model in the Eulerian coordinates with a form of artificial viscosity in [11] have shown that phase transition takes place when initially the density is in the elliptic (or unstable) region; i.e., $u(\cdot, 0) \in\left(u_{1}, u_{2}\right)$. When uniform initial states are considered, their results further lead to a separation, for the total mass, of three ranges corresponding to unstable, metastable, and stable regions. The system also exhibits solutions with multiple thin layers representing phase transitions, and it exhibits that some such configurations move with constant velocity. Existence of periodic traveling wave solutions is proved in a more general setting in [12]. There are also theoretical results on the dynamical stability of phase transitions lying sufficiently close to the Maxwell states on an unbounded domain [9]. The two-dimensional case is studied in [13], where existence of bubble and droplet solutions is proved and their dynamical stability is investigated numerically.

In this paper, we are interested in the stability of the stationary phase transition from $\alpha^{-}$and $\alpha^{+}$, i.e., solutions where $u \sim \alpha^{-}$in one region $\Omega_{-}=\{-a<x<s(t)\}$ and $u \sim \alpha^{+}$in another region $\Omega_{+}=\{s(t)<x<a\}$, whereas $x=s(t)$ is an interface to be determined. Physically, it corresponds to the case that to the left of $x=s(t)$ the material is in the gas phase, whereas to its right, it is in the liquid phase. The interface is located at $x=s(t)$ and is moving (slowly, in the order of $\varepsilon$ ). Using matched asymptotics, we show that the perturbations in $\varepsilon$ order propagate in regions $\Omega_{ \pm}$ according to wave equations with respective wave speed $c^{ \pm}$. The boundary conditions for the wave equations at $\partial \Omega_{ \pm}$are determined from the asymptotic matching. Unlike the infinite domain case where perturbation can escape to spatial infinity in the form of diffusion waves $[5,15]$, the waves propagate back and forth in regions $\Omega_{ \pm}$, thus 
making the interfacial position oscillate around a central point which moves with a certain velocity toward its final destination determined by the mass conservation equation. We shall derive the law of motion for the interface and provide numerical evidence supporting our analysis.

The paper is organized as follows. In section 2 , we perform a matched asymptotic expansion which deduces the wave equations as well as the boundary conditions for the first order perturbations in the outer regions. The solution to the first order equations is studied in section 3. We show the existence and uniqueness of the solution as well as the long-time behavior of the solutions. Results from the numerical simulations are presented in section 4 .

2. Matched asymptotic expansion. In this section, we shall use the matched asymptotic expansion to find approximate solutions to (1.1) and (1.2) with certain layered initial conditions, for sufficiently small positive $\varepsilon$.

2.1. Outline. For the reader's convenience, we first outline our formal matched asymptotic expansion. Details will be given in subsequent subsections.

We divide the interval $[-a, a]$ into three regions: an interfacial region, $[s(t)-$ $\left.2 \varepsilon^{\mu}, s(t)+2 \varepsilon^{\mu}\right]$; two boundary layer regions, $\left[-a,-a+2 \varepsilon^{\mu}\right]$ and $\left[a-2 \varepsilon^{\mu}, a\right]$; and two phase regions, $\left[-a+\varepsilon^{\mu}, s(t)-\varepsilon^{\mu}\right]$ and $\left[s(t)+\varepsilon^{\mu}, a-\varepsilon^{\mu}\right]$. Here $\varepsilon^{\mu}, \mu \in(0,1)$, is a rough estimate of the thickness of the interfacial and boundary layer regions; it of course can be something smaller, say, $\varepsilon \ln ^{2} \varepsilon$.

In the phase regions, we expand the solution in an $\varepsilon$ power series,

$$
\left\{\begin{aligned}
u & \sim u_{0}^{ \pm}+\varepsilon u_{1}^{ \pm}+\varepsilon^{2} u_{2}^{ \pm}+\cdots \\
v & \sim v_{0}^{ \pm}+\varepsilon v_{1}^{ \pm}+\varepsilon^{2} v_{2}^{ \pm}+\cdots
\end{aligned}\right.
$$

where the signs + and - designate to the phase regions $\Omega_{t}^{+}=(s(t), a)$ and $\Omega_{t}^{-}=$ $(-a, s(t))$. We call $(2.1)$ the outer expansion. To reflect that we are dealing with small perturbations from the Maxwell equilibrium states, we fix $u_{0}^{ \pm} \equiv \alpha^{ \pm}$and $v_{0}^{ \pm} \equiv 0$. Substituting the outer expansion into (1.1) and equating the coefficients of $\varepsilon$ powers, we obtain, for each $j$, a system of two differential equations for $\left(u_{j}^{ \pm}, v_{j}^{ \pm}\right)$. These equations are solvable subject to appropriate initial and boundary conditions. For each phase region, there are two boundaries, one at $x=s(t)$ and the other at $x=a$, for the + phase, and one at $x=-a$ for the - phase. Boundary conditions are obtained via boundary layer (at $x= \pm a$ ) and inner (at $x=s(t)$ ) expansions.

At the fixed boundary, say, at $x=-a$, we use the stretched variable $z=(x+a) / \varepsilon$ and expand the solution in $\varepsilon$ power series

$$
\left\{\begin{aligned}
u & \sim u_{0}^{b}+\varepsilon u_{1}^{b}+\varepsilon^{2} u_{2}^{b}+\cdots, \\
v & \sim v_{0}^{b}+\varepsilon v_{1}^{b}+\varepsilon^{2} v_{2}^{b}+\cdots
\end{aligned}\right.
$$

where $u_{i}^{b}, v_{i}^{b}$ are functions of $(z, t)$. Using $\frac{\partial}{\partial x}=\frac{1}{\varepsilon} \frac{\partial}{\partial z}$ we derive from (1.1) systems of differential equations for each $\left(u_{j}^{b}, v_{j}^{b}\right)$. The domain for the equations is $\{(z, t) \mid z \in$ $(0, \infty), t \in(0, \infty)\}$. Boundary conditions at $z=0$ are supplied by substituting $(2.2)$ into (1.2), whereas boundary conditions at $z=\infty$ are obtained by matching the boundary layer and outer expansions. By matching, we mean, after a substitution of $x$ by $-a+\varepsilon z$, that the outer expansion produces exactly the same $\varepsilon$ power series as that of the boundary layer expansion. More precisely, since

$$
\left.u(x, t)\right|_{x=-a+\varepsilon z} \sim \sum_{j=0}^{\infty} \partial_{x}^{j}\left(\sum_{i=0}^{\infty} \varepsilon^{i} u_{i}^{-}(-a, t)\right) \frac{(\varepsilon z)^{j}}{j !} \sim \sum_{k=0}^{\infty} \varepsilon^{k}\left(\sum_{j=0}^{k} \partial_{x}^{j} u_{k-j}^{-}(-a, t) \frac{z^{j}}{j !}\right),
$$


it is required, as $z \rightarrow \infty$, that

$$
\left\{\begin{array}{l}
u_{0}^{b}(z, t)-u_{0}^{-}(-a, t) \rightarrow 0, \\
u_{1}^{b}(z, t)-\left[u_{1}^{-}(-a, t)+\partial_{x} u_{0}^{-}(-a, t) z\right] \rightarrow 0, \\
\cdots \cdots \cdots \cdots \\
u_{j}^{b}(z, t)-\left[u_{j}^{-}(-a, t)+\partial_{x} u_{j-1}(-a, t) z+\cdots+\partial_{x}^{j} u_{0}(-a, t) \frac{z^{j}}{j !}\right] \rightarrow 0, \\
\cdots \cdots \cdots
\end{array}\right.
$$

Similar matching is also required for $v$ and for the + phase.

The boundary layer expansion provides constraints, or boundary conditions, at $x= \pm a$ for the outer expansions; the number of these conditions is down from four as in (1.2) to two, since the remaining two are taken care of by the boundary layers.

In the interfacial region, we use the stretched variable $z=(x-s(t)) / \varepsilon$ and the expansion

$$
\left\{\begin{array}{l}
u=u_{0}^{I}+\varepsilon u_{1}^{I}+\varepsilon^{2} u_{2}^{I}+\cdots, \\
v=v_{0}^{I}+\varepsilon v_{1}^{I}+\varepsilon^{2} v_{2}^{I}+\cdots, \\
s(t)=s_{0}+\varepsilon s_{1}+\varepsilon^{2} s_{2}+\cdots,
\end{array}\right.
$$

where $u_{j}^{I}$ and $v_{j}^{I}$ are functions of $(z, t)$ and $s_{j}$ is a function of $t$, all of them being independent of $\varepsilon$. As before, we can derive from (1.1) systems of equations for each $\left(u_{j}^{I}, v_{j}^{I}\right)$. For each order $j \geq 1$, the system is linear and solvable only if $\dot{s}_{j}$ satisfies a solvability condition. Also, when solvable, the solution is unique up to an additive of a multiple of a special function being the kernel of a linear operator independent of $j$. If we define the interface $x=s(t)$ as the $\alpha^{0}$ level set of the function $u$, the exact amplitude of the additive function can be uniquely determined.

As before, the outer and inner expansions are matched by requiring, as $z \rightarrow \infty$,

$$
\left\{\begin{array}{l}
u_{0}^{I}(z, t) \rightarrow u_{0}^{+}\left(s_{0}, t\right), \\
u_{1}^{I}(z, t)-\left[\partial_{x} u_{0}^{+}\left(s_{0}, t\right)\left(s_{1}+z\right)\right] \rightarrow u_{1}^{+}\left(s_{0}, t\right), \\
u_{2}^{I}(z, t)-\left[\partial_{x} u_{1}^{+}\left(s_{0}, t\right)\left(s_{1}+z\right)+\partial_{x}^{2} u_{0}^{+}\left(s_{0}, t\right)\left(s_{1}+z\right)^{2} / 2+\partial_{x} u_{0}^{+} s_{2}\right] \rightarrow u_{2}^{+}\left(s_{0}, t\right) . \\
\ldots \ldots \ldots
\end{array}\right.
$$

Similar matching is also needed as $z \rightarrow-\infty$ and for $v$. The match provides relations between values of outer expansions from each side of the interface, which can be considered as boundary conditions for the outer expansions at $x=s_{0}(t)$, just completing the systems for each order of outer expansions.

In what follows, we carry out in detail the expansions outlined here.

2.2. Outer expansion. Consider the outer expansion given in (2.1). We are interested in the perturbations around the stationary phase jump between the Maxwell states, so we fix

$$
u_{0}^{ \pm}(x, t)=\alpha^{ \pm}, \quad v_{0}^{ \pm}(x, t)=0 \quad \forall x \in R^{1}, t>0 .
$$

Substituting (2.1) into (1.1) and collecting powers of $\varepsilon$, we obtain, from the coefficients of every power of $\varepsilon$, outer expansion equations for $\left(u_{i}^{ \pm}, v_{i}^{ \pm}\right)$for every $i \geq 0$.

The zeroth order equations are automatically satisfied since $u_{0}^{ \pm}$and $v_{0}^{ \pm}$are constants. The first order expansion equations are

$$
\left\{\begin{array}{l}
u_{1 t}^{ \pm}=v_{1 x}^{ \pm} \\
v_{1}^{ \pm}=\left(c^{ \pm}\right)^{2} u_{1 x}^{ \pm}
\end{array} \quad \forall x \in \Omega_{0}^{ \pm}(t), t>0,\right.
$$


where $c^{ \pm}=\sqrt{-p^{\prime}\left(\alpha^{ \pm}\right)}, \Omega_{0}^{+}(t)=\left(s_{0}(t), a\right), \Omega_{0}^{-}(t)=\left(-a, s_{0}(t)\right)$, and $s_{0}(t)$ is the leading order interface position, to be determined by the inner expansion. Higher order outer expansion equations, for $\left(u_{j}^{ \pm}, v_{j}^{ \pm}\right)$, take the form

$$
\left\{\begin{array}{l}
u_{j}^{ \pm}-v_{j}^{ \pm}=\cdots \\
v_{j}^{ \pm}{ }_{t}-c_{ \pm}^{2} u_{j}^{ \pm}=\cdots
\end{array} \quad \forall x \in \Omega_{0}^{ \pm}(t), \quad t>0, \quad j>1,\right.
$$

where $\cdots$ represents a function depending only on expansions of order $\leq j-1$.

The outer expansion equations (2.7) and (2.8) are wave equations with wave speed $c^{ \pm}$in the corresponding phase region $\Omega_{0}^{ \pm}(t)$. They need supplementary conditions such as initial and boundary conditions. The initial conditions are our choice and are known. The boundary conditions, at $\partial \Omega_{0}^{ \pm}(t)=\left\{ \pm a, s_{0}(t)\right\}$, will be obtained, via matching, from the boundary layer expansions at $x= \pm a$ and the inner expansion at $x=s_{0}(t)$.

2.3. Boundary layer expansions. Due to symmetry, we present in detail only the expansion near $x=-a$. We use the stretched variable $z=\frac{x+a}{\varepsilon}$ and assume that $(u, v)$ possesses the boundary layer expansion (2.2). Substituting (2.2) into (1.1) and collecting powers of $\varepsilon$ gives boundary layer expansion equations for each order of the expansion $\left(u_{j}^{b}, v_{j}^{b}\right), j=0,1, \ldots$

The leading order expansion equations read

$$
\left\{\begin{array}{l}
v_{0 z}^{b}=0 \\
\left(p\left(u_{0}^{b}\right)+u_{0 z z}^{b}-\kappa v_{0 z}^{b}\right)_{z}=0 \quad \forall z \in(0, \infty), t>0 .
\end{array}\right.
$$

An expansion of the boundary condition $u_{x}(-a, t)=v(-a, t)=0$ gives

$$
u_{0 z}^{b}(0, t)=v_{0}^{b}(0, t)=0 \quad \forall t>0 .
$$

The matching condition (2.3) gives, for every $t \geq 0$,

$$
v_{0}^{b}(z, t) \rightarrow 0, \quad u_{0}^{b}(z, t) \rightarrow \alpha^{-} \quad \text { as } z \rightarrow \infty .
$$

Altogether, (2.9)-(2.11) give a unique zeroth order boundary layer expansion

$$
v_{0}^{b}(z, t) \equiv 0, \quad u_{0}^{b}(z, t) \equiv \alpha^{-} \quad \forall z \in[0, \infty), t>0 .
$$

$\left(u_{0}^{b}, v_{0}^{b}\right)$ is trivially determined since the zeroth order outer expansion has been fixed.

At the next order, the boundary layer expansion equations read

$$
\left\{\begin{array}{l}
v_{1 z}^{b}=0 \\
\left(u_{1 z z}^{b}-c_{-}^{2} u_{1}^{b}-\kappa v_{1 z}^{b}\right)_{z}=0
\end{array} \quad \forall z \in(0, \infty), t>0 .\right.
$$

Expansion of the boundary condition (1.2) and the matching with outer expansion give

$$
\left\{\begin{array} { l } 
{ v _ { 1 } ^ { b } ( 0 , t ) = 0 , } \\
{ u _ { 1 z } ^ { b } ( 0 , t ) = 0 , }
\end{array} \quad \left\{\begin{array}{l}
\lim _{z \rightarrow \infty} v_{1}^{b}(z)=v_{1}^{-}(-a, t) \\
\lim _{z \rightarrow \infty} u_{1}^{b}(z, t)=u_{1}^{-}(-a, t)
\end{array} \quad \forall t>0 .\right.\right.
$$

Clearly, in order to obtain a solution $v_{b}^{1}$, it is necessary and sufficiently to have

$$
v_{1}^{-}(-a, t)=0 \quad \forall t>0 .
$$


With this condition, the unique solution of the first order boundary layer expansion is

$$
v_{1}^{b}(z, t) \equiv 0, \quad u_{1}^{b}(z, t)=u_{1}^{-}(-a, t) \quad \forall z \geq 0, t>0 .
$$

We remark that the boundary condition $v(-a, t)=0$ gives a restriction $v_{1}^{-}(-a, 0)$ $=0$ to the outer expansion, in contrast to $u_{x}(-a, t)=0$, which gives no restriction to the outer expansion. This is due to the viscous regularization term $\left(\varepsilon^{2} u_{x x}\right)_{x}$ in (1.1).

For each $j \geq 2$, the $j$ th order boundary layer expansion equations read

$$
\left\{\begin{array}{l}
\left(v_{j}^{b}\right)_{z}=\cdots \\
\left(\left(u_{j}^{b}\right)_{z z}-\left(c^{-}\right)^{2} u_{j}^{b}-\kappa v_{j}^{b}\right)_{z}=\cdots
\end{array} \quad \forall z \in(0, \infty), t>0 .\right.
$$

The boundary and matching conditions are

$$
\left\{\begin{array} { l } 
{ v _ { j } ^ { b } ( 0 , t ) = 0 , } \\
{ ( u _ { j } ^ { b } ) _ { z } ( 0 , t ) = 0 , }
\end{array} \quad \left\{\begin{array}{l}
\lim _{z \rightarrow \infty}\left(v_{j}^{b}(z, t)-\cdots\right)=v_{j}^{-}(-a, t) \\
\lim _{z \rightarrow \infty}\left(u_{b}^{j}(z, t)-\cdots\right)=u_{j}^{-}(-a, t)
\end{array} \quad \forall t>0 .\right.\right.
$$

Here $\cdots$ are polynomials of order no larger than $j-1$ and depend only on expansions of order $\leq j-1$. Integrating equation for $v_{j}^{b}$, one sees that for the $j$ th order expansion to have a solution, the outer expansion $v_{b}^{-}$must satisfy $v_{j}^{-}(-a, t)=\cdots$, where "..." is a quantity that can be calculated from the lower order expansion. In addition, with this constraint on $v_{j}^{-}(-a, t)$, there is a unique solution $\left(v_{j}^{b}, u_{j}^{b}\right)$ for each value of $u_{j}^{-}(-a, t)$.

Similarly, one can perform the boundary layer expansion at the right boundary $x=a$.

We summarize the boundary layer expansion as follows:

(1) There is a unique zeroth order expansion that matches the zeroth order outer expansion (2.6).

(2) For each $j \geq 1$, there are functions $g_{j}^{-}(t)$ and $g_{j}^{+}(t)$, which are uniquely determined by expansions of order $\leq j-1$, such that the $j$ th order boundary layer expansion equations have a solution if and only if the outer $j$ th order expansion $\left(v_{j}^{ \pm}, u_{j}^{ \pm}\right)$satisfies the boundary condition

$$
v_{j}^{-}(-a, t)=g_{j}^{-}(t), \quad v_{j}^{+}(a, t)=g_{j}^{+}(t) \quad \forall t>0 .
$$

When $j=1$, calculation shows that $g_{1}^{+}(t)=g^{-}(t)_{1} \equiv 0$.

(3) If the solvability condition (2.14) is satisfied, then for any given boundary value $u_{j}^{ \pm}( \pm a, t)$, there is a unique solution $\left(u_{j}^{b}, v_{j}^{b}\right)$ to the $j$ th order boundary layer expansion.

In short, the boundary layer expansion provides the boundary conditions for the outer expansion equations (2.7) and (2.8).

2.4. Inner layer expansion. Near the interface $x=s(t)$, we use the stretched variable $z=\frac{x-s(t)}{\varepsilon}$ and assume that the solution $(u, v)$ has the inner expansion of the form (2.4). Substituting (2.4) into (1.1), we need to have

$$
\begin{aligned}
\Sigma_{j} \varepsilon^{j} u_{j t}^{I}-\frac{1}{\varepsilon}\left(\Sigma_{j} \varepsilon^{j} \dot{s}_{j}\right)\left(\Sigma_{j} \varepsilon^{j} u_{j z}^{I}\right) & \sim \frac{1}{\varepsilon} \Sigma_{j} \varepsilon^{j} v_{j}^{I}, \\
\Sigma_{j} \varepsilon^{j} v_{j t}^{I}-\frac{1}{\varepsilon}\left(\Sigma_{j} \varepsilon^{j} \dot{s}_{j}\right)\left(\Sigma_{j} \varepsilon^{j}\right) v_{j z}^{I} & \sim \frac{1}{\varepsilon} \frac{\partial}{\partial z}\left\{p\left(\Sigma_{j} \varepsilon^{j} u_{j}^{I}\right)+\left(\Sigma_{j} \varepsilon^{j} u_{j}^{I}\right)_{z z}-\kappa \Sigma_{j} \varepsilon^{j} v_{j z}^{I}\right\} .
\end{aligned}
$$


Expanding and collecting powers of $\varepsilon$ we then obtain inner expansion equations for each order $j=0,1, \ldots$

The leading order inner expansion equations are

$$
\left\{\begin{array}{l}
-\dot{s}_{0} u_{0 z}^{I}=v_{0 z}^{I} \\
-\dot{s}_{0} v_{0 z}^{I}=\left\{p\left(u_{0}^{I}\right)+u_{0 z z}^{I}-\kappa v_{0 z}^{I}\right\}_{z}
\end{array} \quad \forall z \in R^{1}, t>0 .\right.
$$

To match the outer expansion, we need, for each $t>0$,

$$
v_{0}^{I}(z, t) \rightarrow 0, \quad u_{0}^{I}(z, t) \rightarrow \alpha^{ \pm} \quad \text { as } \quad z \rightarrow \pm \infty .
$$

With such a requirement, the only solution to the first equation in (2.15) is

$$
\dot{s}_{0}(t)=0, \quad v_{0}^{I}(z, t)=0 \quad \forall z \in R^{1}, t>0 .
$$

We remark that $\dot{s}_{0} \equiv 0$ is expected since our perturbation from the Maxwell equilibrium states is of $\varepsilon$ order.

With (2.16), the second equation in (2.15) and its associated boundary condition give

$$
\left\{\begin{array}{l}
u_{0 z z}^{I}+p\left(u_{0}^{I}\right)=Q^{*} \\
u_{0}^{I}( \pm \infty, t)=\alpha^{ \pm}
\end{array} \quad \forall z \in R^{1}, t>0,\right.
$$

where $Q^{*}$ is an integration constant. Clearly, to ensure the existence of a solution, $\alpha^{+}$and $\alpha^{-}$must be the Maxwell states; namely, (1.5) must hold. It is easy to see that with (1.4) and (1.5), (2.17) has a solution, unique up to a translation in $z$. To modulo the translation invariance, we assume that the interface $x=s(t)$ is indeed defined as the $\alpha^{0}$ level set of $u$, i.e., at $z=0, u=\alpha^{0}$. This gives the extra constraint $u_{0}^{I}(0, t)=\alpha^{0}$, and therefore it determines a unique $u_{0}^{I}$.

In summary, there is a unique leading order inner expansion given by

$$
s_{0}(t) \equiv s_{0}(0), \quad v_{0}^{I}(z, t) \equiv 0, \quad u_{0}^{I}(z, t)=U(z) \quad \forall z \in R^{1}, t>0,
$$

where $U$ is the unique solution to

$$
\left\{\begin{array}{l}
U^{\prime \prime}(z)+p(U(z))=Q^{*}\left(:=p\left(\alpha^{ \pm}\right)=p\left(\alpha^{0}\right)\right), \\
U( \pm \infty)=\alpha^{ \pm}, U(0)=\alpha^{0} .
\end{array}\right.
$$

Observe that $(u:=U(x / \varepsilon), v:=0)$ is the steady state of (1.1) in $R^{1}$. From (2.18), one sees that in leading order, the transition profile from $u \sim \alpha^{-}$phase to $u \sim \alpha^{+}$ phase is independent of time and is given by the Maxwell connection profile $U$ with an $\varepsilon$ scaling.

With the substitution of the leading order inner expansion, the next order (first order) inner expansion equations are

$$
\left\{\begin{array}{l}
-\dot{s}_{1} U(z)_{z}=v_{1 z}^{I} \\
\left\{p^{\prime}(U) u_{1}^{I}+u_{1 z z}^{I}-\kappa v_{1 z}^{I}\right\}_{z}=0
\end{array} \quad \forall z \in R^{1}, t \geq 0 .\right.
$$

The matching of inner and outer expansion reads

$$
u_{1}^{I}(z, t) \rightarrow u_{1}^{ \pm}\left(s_{0}, t\right), \quad v_{1}^{I}(z, t) \rightarrow v^{ \pm}\left(s_{0}, t\right) \quad \text { as } z \rightarrow \pm \infty .
$$


To ensure the existence of $\left(u_{1}^{I}, v_{1}^{I}\right)$ with the required behavior at $z= \pm \infty$, we need a few solvability conditions.

A pair of solvability conditions are obtained immediately by integrating the two equations in (2.20) over $R$ :

$$
\begin{aligned}
-\dot{s}_{1}\left(\alpha^{+}-\alpha^{-}\right) & =v_{1}^{+}\left(s_{0}, t\right)-v_{1}^{-}\left(s_{0}, t\right), \\
p^{\prime}\left(\alpha^{+}\right) u_{1}^{+}\left(s_{0}, t\right) & =p^{\prime}\left(\alpha^{-}\right) u_{1}^{-}\left(s_{0}, t\right) .
\end{aligned}
$$

To find the remaining solvability condition(s), we begin to solve (2.20), (2.21). There is a unique solution for $v_{1}^{I}$ given by

$$
v_{1}^{I}(z, t)=v^{-}\left(s_{0}, t\right)-\dot{s}_{1}(t)\left(U(z)-\alpha^{-}\right) \quad \forall z \in R^{1}, t>0 .
$$

Substituting it into the second equation in (2.20) and integrating the resulting equation from $-\infty$ to $z$, we obtain

$$
\mathcal{L}\left(u_{1}^{I}\right):=p^{\prime}(U) u_{1}^{I}+u_{1 z z}^{I}=p^{\prime}\left(\alpha^{-}\right) u_{1}^{-}\left(s_{0}, t\right)+\dot{s}_{1} \kappa U_{z} .
$$

Since $\mathcal{L}$ has a zero eigenvalue with kernel $U_{z}$ (as $\left.\mathcal{L}\left(U_{z}\right)=0\right)$, the above equation has a solution if and only if the right-hand side is orthogonal to $U_{z}$ in $L^{2}(R)$. (This condition can be seen more directly by multiplying both side of the equation by $U_{z}$ and integrating the resulting equation over $R$.) That $U_{z} \perp p^{\prime}\left(\alpha^{-}\right) u_{1}^{-}\left(s_{0}, t\right)+\kappa \dot{s}_{1} U_{z}$ is equivalent to

$$
\dot{s}_{1}=\frac{-\left(c^{-}\right)^{2} u_{1}^{-}\left(s_{0}, t\right)\left(\alpha^{+}-\alpha^{-}\right)}{\kappa \int_{\infty}^{\infty}\left(U_{z}\right)^{2} d z}
$$

One can eliminate $\dot{s}_{1}$ from (2.22) to obtain self-contained boundary conditions for the outer expansion $\left(u_{1}^{ \pm}, v_{1}^{ \pm}\right)$at $x=s_{0}$ :

$$
v_{1}^{+}\left(s_{0}, t\right)-v^{-}\left(s_{0}, t\right)=\sigma\left(c^{+}\right)^{2} u^{+}\left(s_{0}, t\right)=\sigma\left(c^{-}\right)^{2} u^{-}\left(s_{0}, t\right) \quad \forall t>0,
$$

where

$$
\sigma:=\frac{\left(\alpha^{+}-\alpha^{-}\right)^{2}}{\int_{R} U_{z}^{2}(z) d z}=\frac{\left(\alpha^{+}-\alpha^{-}\right)^{2}}{\int_{\alpha^{-}}^{\alpha^{+}} \sqrt{2 P(s)} d s .}, \quad P(u):=\int_{\alpha^{-}}^{u}\left[p\left(\alpha^{-}\right)-p(s)\right] d s .
$$

Now (2.26), together with $v_{1}^{ \pm}( \pm a, t)=0$ obtained from the boundary layer expansion, provides the exact number of boundary conditions needed by the outer expansion equations (2.7). With given initial values of our choice, we can uniquely solve the first order outer expansion $\left(u_{1}^{ \pm}, v_{1}^{ \pm}\right)$. (See the next section for more details.)

Once we have $\left(u_{1}^{ \pm}, v_{1}^{ \pm}\right)$, we can use (2.25) to determine $s_{1}$ and use (2.24) to find $u_{1}^{I}$. There are infinitely many solutions of $u_{1}^{I}$, all being a special solution plus a multiple of $U_{z}$, since $\mathcal{L}\left(U_{z}\right)=0$; nevertheless, a unique multiple can be found by the constraint $u_{1}^{I}\left(s_{0}, 0\right)=0$, since we define $x=s(t)$ as the $\alpha^{0}$ level set of $u$. This determined a unique $u_{1}^{I}$. Knowing $u^{ \pm}( \pm a, t)$, the first order boundary layer expansion can also be determined. Therefore, we obtain completely all the first order expansion functions $u_{1}^{ \pm}, s_{1}, u_{1}^{I}, u_{1}^{b}$.

The general $j$ th $(j \geq 2)$ order inner expansion equations read

$$
\left\{\begin{array}{l}
\left(\dot{s}_{j} u_{j}^{I}-v_{j}^{I}\right)_{z}=\cdots \\
\left(\mathcal{L}\left(u_{j}^{I}\right)-\kappa v_{j z}^{I}\right)_{z}=\cdots
\end{array} \quad \forall z \in R, t>0 .\right.
$$


The interface and matching conditions are

$u_{j}^{I}(0, t)=0, \quad \lim _{z \rightarrow \pm \infty}\left(u_{j}^{I}(z, t)-\cdots\right)=u_{j}^{ \pm}\left(s_{0}, t\right), \quad \lim _{z \rightarrow \pm \infty}\left(v_{j}^{I}(z, t)-\cdots\right)=v_{j}^{ \pm}\left(s_{0}, t\right)$.

Here $\cdots$ are functions depending only on expansions of order $\leq j-1$.

To ensure the existence of a unique solution $\left(u_{j}^{I}, v_{j}^{I}\right)$, the boundary value $\left(u_{j}^{ \pm}\left(s_{0}, t\right)\right.$, $\left.v_{j}^{ \pm}\left(s_{0}, t\right)\right)$ of the $j$ th order outer expansion at $x=s_{0}$ needs to satisfy the solvability conditions (for inner expansion)

$$
\left(c^{+}\right)^{2} u_{j}^{+}\left(s_{0}, t\right)-\left(c^{-}\right)^{2} u_{j}^{-}\left(s_{0}, t\right)=\cdots, \quad \dot{s}_{j}\left[\alpha^{+}-\alpha^{-}\right]+\left[v_{j}^{+}\left(s_{0}, t\right)-v^{-}\left(s_{0}, t\right)\right]=\cdots,
$$

and the $j$ th order interface position expansion $\dot{s}_{j}$ needs to satisfy

$$
\dot{s}_{j}=\frac{p^{\prime}\left(\alpha^{-}\right) u_{j}^{-}\left(s_{0}, t\right)\left(\alpha^{+}-\alpha^{-}\right)}{\kappa \int_{\infty}^{\infty}\left(U_{z}\right)^{2} d z}+\cdots
$$

Here again, $\cdots$ represents known functions if lower order expansions are found.

One can eliminate $\dot{s}_{j}$ from (2.28) and (2.29) to obtain self-contained boundary conditions for $\left(u_{j}^{ \pm}, v_{j}^{ \pm}\right)$at $x=s_{0}$ :

$$
v_{j}^{+}\left(s_{0}, t\right)-v_{j}^{-}\left(s_{0}, t\right)=\sigma\left(c^{+}\right)^{2} u_{j}^{+}\left(s_{0}, t\right)+\cdots=\sigma\left(c^{+}\right)^{2} v_{j}^{-}\left(s_{0}, t\right)+\cdots \quad \forall t>0 .
$$

With these two conditions and the boundary conditions (2.14), we can solve uniquely the $j$ th order outer expansion $\left(u_{j}^{ \pm}, v_{j}^{ \pm}\right)$from (2.8) after we provide initial conditions for them. Once we find the outer expansion of $j$ th order, we can determined uniquely all the other $j$ th order expansions and, if necessary, go on to the next order expansion.

2.5. The approximate solution. Let $k \geq 1$ be an integer representing the order of approximation we need. For $j$ from 0 to $k$, we use the matched asymptotic expansion aforementioned to find successively the outer, interface position, inner, and boundary layer expansions. We take the finite sums

$$
\left\{\begin{array}{l}
u^{b}(z, t)=\sum_{j=0}^{k} \varepsilon^{j} u_{j}^{b}{ }^{\prime} \\
u_{ \pm}^{o}(x, t)=\sum_{j=1}^{k} \varepsilon^{j} u_{j}^{ \pm} \\
u^{I}(z, t)=\sum_{j=1}^{k} \varepsilon^{j} u_{j}^{I}, \\
S(t)=\sum_{j=1}^{k} \varepsilon^{j} s_{j}
\end{array}\right.
$$

and similar sums for $v$.

Since $u^{b}, u_{ \pm}^{o}$, and $u^{I}$ match in their overlap regions, we can glue them to obtain a $k$ th order approximation solution. For example, we can glue $u_{+}^{o}$ and $u^{I}$ via

$$
\zeta\left(\frac{x-S(t)}{\varepsilon^{\mu}}\right) u_{+}^{o}(x, t)+\left.\left[1-\zeta\left(\frac{x-S(t)}{\varepsilon^{\mu}}\right)\right] u^{I}(z, t)\right|_{z=(x-S(t)) / \varepsilon}, \quad \varepsilon^{\mu} \leq x-S(t) \leq 2 \varepsilon^{\mu},
$$

where $\zeta(s)$ is a cutoff function satisfying

$$
\zeta(s)=1 \text { if } s \geq 2 \text { and } \zeta(s)=0 \text { if } s<1 .
$$

We believe that the approximate solution so constructed approximates the true solution. We expect that rigorous verification can be made by employing the method used for the verification of matched asymptotic expansions in any space dimensions by Alikakos, Bates, and Chen [1] for the Cahn-Hilliard equation, by Caginalp and Chen [2] for the phase field equations, and by de Mottoni and Schatzman [7] for the Allen-Cahn equation. The method was recently summarized by Chen in [4]. 
3. Solutions to the first order expansion. Since we are interested in the effect of small initial perturbation of (1.1) from its steady state and also the boundary effect, in this section we study in detail the solutions to our first order formal expansion.

We collect the equations for $\left(u_{1}^{ \pm}, v_{1}^{ \pm}\right)$:

$$
\begin{cases}u_{1 t}^{ \pm}=v_{1 x}^{ \pm}, & x \in \Omega_{0}^{ \pm}, t>0, \\ v_{1 t}^{ \pm}=\left(c^{ \pm}\right)^{2} u_{1 x}^{ \pm}, & x \in \Omega_{0}^{ \pm}, t>0, \\ v_{1}^{+}\left(s_{0}, t\right)-v_{1}^{-}\left(s_{0}, t\right)=\sigma\left(c^{ \pm}\right)^{2} u_{1}^{ \pm}\left(s_{0}, t\right), & \\ v_{1}^{+}(a, t)=v_{1}^{-}(-a, t)=0, & \\ u_{1}(x, 0)=u_{0}(x), v_{1}^{ \pm}(x, 0)=v_{0}^{ \pm}(x), & x \in \Omega_{0}^{ \pm},\end{cases}
$$

where $\Omega_{0}^{+}=\left(s_{0}, a\right), \Omega_{0}^{-}=\left(-a, s_{0}\right), s_{0} \in(-a, a)$ is a fixed constant, and $\sigma$ is as in (2.27).

Once we find $\left(u_{1}^{ \pm}, v_{1}^{ \pm}\right)$, the first order perturbation of interface position function $s_{1}(t)$ can be obtained by integrating the ODE

$$
\dot{s}_{1}(t)=\frac{\sigma\left(c^{-}\right)^{2} u_{1}^{-}\left(s_{0}, t\right)}{\alpha^{-}-\alpha^{+}} \quad\left(=\frac{\sigma\left(c^{+}\right)^{2} u_{1}^{+}\left(s_{0}, t\right)}{\alpha^{-}-\alpha^{+}}\right) .
$$

Although (P1) is a linear problem, we find that the problem is nonstandard, and the phenomenon that the solution provides is quite rich. Hence, we shall use three different but quite elementary methods to study problem (P1):

(i) an energy method to give uniqueness and asymptotic behavior of solutions;

(ii) a constructive method to provide the existence of solutions;

(iii) a separation of variables method to supplement more detailed behavior of the solution.

For convenience, we define functions

$$
c(x)=\left\{\begin{array}{ll}
c^{+} & x \in\left(s_{0}, a\right], \\
c^{-} & x \in\left[-a, s_{0}\right),
\end{array} \quad\left(u_{1}, v_{1}\right)= \begin{cases}\left(u_{1}^{+}, v_{1}^{+}\right) & x \in\left(s_{0}, a\right], t \geq 0 \\
\left(u^{-}, v^{-}\right) & x \in\left[-a, s_{0}\right), t \geq 0 .\end{cases}\right.
$$

As $\left(c^{ \pm}\right)^{2} u_{1}^{ \pm}$is continuous across $x=s_{0}$, we denote the common value at $x=s_{0}$ by $c^{2} u_{1}$.

3.1. Energy estimates. Denote $\Omega=\left(-a, s_{0}\right) \cup\left(s_{0}, a\right)$. For solutions of (P1), we can calculate

$$
\begin{aligned}
\frac{d}{d t} \int_{-a}^{a}\left(c^{2} u_{1}^{2}+v_{1}^{2}\right) d x & =2 \int_{\Omega}\left(c^{2} u_{1} u_{1 t}+v_{1} v_{1 t}\right)=2 \int_{\Omega}\left(c^{2} u_{1} v_{1 x}+c^{2} v_{1} u_{1 x}\right) \\
& =\left[c^{2} u_{1} v_{1}\right]_{s^{0}+}^{s^{0}}=-2 \sigma\left(c^{2} u_{1}\left(s_{0}, t\right)\right)^{2}, \\
\frac{d}{d t} \int_{\Omega}\left(c^{4} u_{1 x}^{2}+c^{2} v_{1 x}^{2}\right) d x & =2 \int_{\Omega}\left(c^{4} u_{1 x} u_{1 t x}+c^{2} v_{1 x} v_{1 x t}\right)=2 \int_{\Omega}\left(c^{2} v_{1 t} u_{1 t x}+c^{2} u_{1 t} v_{1 x t}\right) \\
& =c^{2} u_{1 t}\left[v_{1 t}\right]_{s_{0}+}^{s_{0}-}=-\left(c^{2} u_{1 t}\left(s_{0}, t\right)\right)^{2}, \\
\frac{d}{d t} \int_{\Omega} u_{1} d x & =\int_{\Omega} v_{1 x} d x=-\left[v_{1}\right]_{s_{0}^{-}}^{s^{0}+}=-\left(\alpha^{+}-\alpha^{-}\right) \dot{s}_{1}(t) .
\end{aligned}
$$

Integration over $t$ then gives, for every $t>0$, 


$$
\int_{-a}^{a}\left(c^{2} u_{1}^{2}+v_{1}^{2}\right)(x, t) d x+2 \int_{0}^{t}\left(c^{2} u_{1}\left(s_{0}, \tau\right)\right)^{2} d \tau=\int_{-a}^{a}\left(c^{2} u_{1}^{2}+v_{1}^{2}\right)(x, 0) d x,
$$

$$
\begin{aligned}
\int_{\Omega}\left(c^{4} u_{1 x}^{2}+c^{2} v_{1 x}^{2}\right)(x, t) d x+2 \int_{0}^{t}\left(c^{2} u_{1 t}\left(s_{0}, \tau\right)\right)^{2} d \tau & =\int_{\Omega}\left(c^{2} u_{1 x}^{2}+v_{1 x}^{2}\right), \\
(3.5) \quad\left(\alpha^{+}-\alpha^{-}\right) s_{1}(t)+\int_{-a}^{a} u_{1}(x, t) d t & =\left(\alpha^{+}-\alpha^{-}\right) s_{1}(0)+\int_{-a}^{a} u_{1}(x, 0) d x .
\end{aligned}
$$

With these energy identities, we can now prove the following.

TheOrem 3.1. Assume that $u_{0}, v_{0} \in H^{1}(\Omega)$. Then (P1) has at most one solution. In addition, if there is a solution, the solution satisfies

$$
\begin{aligned}
\lim _{t \rightarrow \infty} c^{2} u_{1}\left(s_{0}, t\right) & =0 \\
\lim _{t \rightarrow \infty} s_{1}(t) & =s_{1}(0)+\frac{1}{\alpha^{+}-\alpha^{-}} \int_{-a}^{a} u_{0}(x) d x .
\end{aligned}
$$

Existence of a solution will be given in the next subsection.

Proof. The uniqueness follows from the linearity of problem (P1) and the first energy identity (3.3). (With an appropriate definition of weak solutions, one can actually show that weak solutions are unique if initial data is only in $L^{2}$.)

Next we prove (3.6). From (3.3) and (3.4),

$$
\int_{0}^{\infty}\left[\left(c^{2} u_{1}\left(s_{0}, t\right)\right)^{2}+\left(c^{2} u_{1 t}\left(s_{0}, t\right)\right)^{2}\right] d t<\infty .
$$

It then follows that for any $t_{2}>t_{1} \geq 0$,

$$
\begin{aligned}
\left|\left(c^{2} u_{1}\left(s_{0}, t_{2}\right)\right)^{2}-\left(c^{2} u_{1}\left(s_{0}, t_{1}\right)\right)^{2}\right| & \leq 2 \int_{t_{1}}^{t_{2}}\left|c^{2} u_{1}\right|\left|c^{2} u_{1 t}\right|\left(s_{0}, \tau\right) d \tau \\
& \left.\leq \int_{t_{1}}^{\infty}\left\{\left(c^{2} u_{1}\right)^{2}+\left(c^{2} u_{1 t}\right)^{2}\right)\left(s_{0}, \tau\right)\right\} d \tau
\end{aligned}
$$

This implies that $\lim _{t \rightarrow \infty} c^{2} u_{1}\left(s_{0}, t\right)$ exists. As $\int_{0}^{\infty}\left(c^{2} u_{1}\left(s_{0}, t\right)\right)^{2} d t$ is finite, this limit must be zero. This proves (3.6).

Next we prove (3.7). From the mass conservation (3.5), we need only to show that

$$
\lim _{t \rightarrow \infty} \int_{-a}^{a} u_{1}(\cdot, t)=0
$$

To this end, we need a few steps.

Since $\sup _{t \geq 0}\left\|u_{1}(\cdot, t), v_{1}(\cdot, t)\right\|_{H^{1}(\Omega)}$ is bounded, the $\omega$-limit set of $\{(u(\cdot, t), v(\cdot, t))\}_{t>0}$ is nonempty. Let $\left(\mathbf{u}_{0}, \hat{v}_{0}\right)$ be any point in the $\omega$-limit set; namely, there exists a sequence $\left\{t_{j}\right\}_{j=1}^{\infty}$ such that as $j \rightarrow \infty, t_{j} \rightarrow \infty$ and $\left(u_{1}\left(\cdot, t_{j}\right), v_{1}\left(\cdot, t_{j}\right)\right) \rightarrow\left(\mathbf{u}_{0}, \hat{v}_{0}\right)$ strongly in $L^{2}((-a, a))$ and weakly in $H^{1}(\Omega)$.

Consider the initial value problem (P1) with initial value $\left(\mathbf{u}_{0}, \hat{v}_{0}\right)$. Denote the solution by $(\mathbf{u}, \hat{v})$ (existence to be shown in the next subsection). Then as $\left(u_{1}\left(\cdot, t_{j}\right), v_{1}\left(\cdot, t_{j}\right)\right)$ 
$\rightarrow(\mathbf{u}(\cdot, 0), \mathbf{u}(\cdot, 0))$, applying $(3.3)$ to the difference between $\left(u_{1}\left(\cdot, t_{j}+\cdot\right), v_{1}\left(\cdot, t_{j}+\cdot\right)\right)$ and $(\mathbf{u}, \hat{v})$, we obtain

$\lim _{j \rightarrow \infty}\left\{\sup _{t>0} \int_{-a}^{a}\left[c^{2}\left(\mathbf{u}(\cdot, t)-u_{1}\left(\cdot, t_{j}+t\right)\right)\right]^{2}+\int_{0}^{\infty}\left[c^{2}\left(\mathbf{u}\left(s_{0}, t\right)-u_{1}\left(s_{0}, t_{j}+t\right)\right)\right]^{2} d t\right\}=0$.

It then follows from (3.6) that

$$
\mathbf{u}\left(s_{0}-, t\right)=\mathbf{u}\left(s_{0}+, t\right) \equiv 0 \quad \forall t \geq 0 .
$$

With this boundary condition, we then can completely solve for $(\mathbf{u}, \hat{v})$ from the wave equations in $\left(-a, s_{0}\right) \times(0, \infty)$ and $\left(s_{0}, a\right) \times(0, \infty)$ separately. The resulting solution must be continuous across $x=s_{0}$ since $\mathbf{u}\left(s_{0} \pm, t\right)=0$ and $\left[\hat{v}\left(s_{0}, t\right)\right]_{s_{0}-}^{s_{0}+}=c^{2} \mathbf{u}\left(s_{0}, t\right)=0$. Consequently, $(\mathbf{u}, \hat{v})$ solves the wave equations on $(-a, a) \times(0, \infty)$.

In terms of Fourier's expansion, $(\mathbf{u}, \hat{v})$ can be written as a superposition of waves of the form $\left(e^{\lambda t} \phi(x, \lambda), e^{\lambda} \psi(x, \lambda)\right)$, where $(\phi, \psi, \lambda)$ solves the eigenvalue problem

$$
\psi^{\prime}=\lambda \phi, \quad c^{2} \phi^{\prime}=\lambda^{2} \psi, \quad x \in(-a, a)
$$

with boundary conditions $\psi(-a)=\psi(a)=0$. Observe that every eigenfunction of nonzero eigenvalue satisfies $\int_{-a}^{a} \phi d x=\frac{1}{\lambda} \int_{-a}^{a} \psi^{\prime}=0$. Also, when $\lambda=0$, the only solution is $\psi \equiv 0$ and $\phi=$ constant. As $\mathbf{u}\left(s_{0}, t\right) \equiv 0$, in the superposition of $\mathbf{u}$, the special eigenfunction $(\phi=$ const, $\psi \equiv 0, \lambda=0)$ is not contained. Hence $\int_{-a}^{a} \mathbf{u}(x, t) d x=0 \forall t \geq 0$. In view of (3.9), we obtain (3.8), which in turn, by (3.5), implies (3.7). This completes the proof.

3.2. Construction of the solution. The general solution to the PDEs in (P1) is given by

$$
\left\{\begin{array}{l}
u_{1}^{ \pm}(x, t)=F\left( \pm c^{ \pm} t+x-s_{0}\right)+G\left( \pm c^{ \pm} t \pm a-x\right), \\
v_{1}^{ \pm}(x, t)= \pm c^{ \pm}\left[F\left( \pm c^{ \pm} t+x-s_{0}\right)-G\left( \pm c^{ \pm} t \pm a-x\right)\right],
\end{array} \quad x \in I_{0}^{ \pm}, t \geq 0 .\right.
$$

Note that to find $\left(u_{1}^{+}, v_{1}^{+}\right)$, we need only find $(F(\xi), G(\xi)) \forall \xi>0$, and to find $\left(u_{1}^{-}, v_{1}^{-}\right)$, we need only find $(F(\xi), G(\xi)) \forall \xi<0$. Altogether, we need only find $(F(\xi), G(\xi)) \forall \xi \in R$.

The initial condition gives the definition of $F, G$ for $\xi \in\left(-a-s_{0}, a-s_{0}\right)$ :

$$
\left\{\begin{array}{l}
F(\xi)=\frac{1}{2}\left[u_{0}\left(s_{0}+\xi\right)+v_{0}\left(s_{0}+\xi\right) / c^{ \pm}\right], \\
G(\xi)=\frac{1}{2}\left[u_{0}( \pm a-\xi)+v_{0}( \pm a-\xi) / c^{ \pm}\right],
\end{array} \quad \pm \xi \in\left(0, a \mp s_{0}\right) .\right.
$$

The boundary condition $v_{1}^{ \pm}( \pm a, t)=0$ gives the relation

$$
F\left( \pm \xi \pm a-s_{0}\right)=G( \pm \xi) \quad \forall \xi>0 .
$$

This relation allows us to define $F$ in $\left(-l_{1}-\left(a+s_{0}\right),-\left(a+s_{0}\right)\right) \cup\left(a-s_{0}, l_{2}+a-s_{0}\right)$, as long as we know the value of $G$ in interval $\left(-l_{1}, l_{2}\right)$, where $l_{1}$ and $l_{2}$ can be any positive constants.

The interface condition $\sigma\left(c^{+}\right)^{2} u_{1}^{+}\left(s_{0}, t\right)=\sigma\left(c^{-}\right)^{2} u_{1}^{-}\left(s_{0}, t\right)=v_{1}^{+}\left(s_{0}, t\right)-v_{1}^{-}\left(s_{0}, t\right)$ gives

$$
\begin{aligned}
& \sigma\left(c^{+}\right)^{2}\left[F\left(c^{+} t\right)+G\left(c^{+} t+a-s_{0}\right)\right]=\sigma\left(c^{-}\right)^{2}\left[F\left(-c^{-} t\right)+G\left(-c^{-} t-a-s_{0}\right)\right] \\
& \quad=\left[c^{+} F\left(c^{+} t\right)+c^{-} F\left(-c^{-} t\right)\right]-\left[c^{+} G\left(c^{+} t+a-s_{0}\right)+c^{-} G\left(-c^{-} t-a-s_{0}\right)\right]=: Q(t) .
\end{aligned}
$$


Solving this system gives

$$
\begin{aligned}
& G\left( \pm \xi \pm a-s_{0}\right)=\frac{2\left[c^{+} F\left(c^{+} \xi / c^{ \pm}\right)+c^{-} F\left(-c^{-} \xi / c^{ \pm}\right)\right]}{\left(c^{ \pm}\right)^{2}\left(\sigma+1 / c^{+}+1 / c^{-}\right)}-F( \pm \xi) \quad \forall \xi>0 \\
& \dot{s}_{1}(t)=\frac{Q(t)}{\alpha^{+}-\alpha^{-}}=\frac{2 \sigma\left[c^{+} F\left(c^{+} t\right)+c^{-} F\left(-c^{-} t\right)\right]}{\left(\alpha^{-}-\alpha^{+}\right)\left(\sigma+1 / c^{+}+1 / c^{-}\right)} \quad \forall t>0 .
\end{aligned}
$$

One can use an induction argument to show that (3.10), (3.11), and (3.12) uniquely define $(F(\xi), G(\xi)) \forall \xi \in R^{1}$, thereby proving the following existence theorem.

TheOREM 3.2. For every $\left(u_{0}, v_{0}\right) \in H^{1}(\Omega)$, problem $(\mathrm{P} 1)$ admits a unique solution.

Though we have an explicit solution, it is hard to see its asymptotic behavior, as $t \rightarrow \infty$, such as those provided in Theorem 3.1. Nevertheless, in some special cases, it can be quite useful. Consider, for example, the special case when

$$
\left(a-s_{0}\right) / c^{+}=\left(a+s_{0}\right) / c^{-}=: T .
$$

Namely, the sound waves in the $\alpha^{+}$phase and $\alpha^{-}$phase take the same amount of time $T$ to travel from the interface $\left(x=s_{0}\right)$ to the container's boundary $(x= \pm a)$.

Under assumption (3.14), we can directly verify, for every $t>0$,

$$
Q(t+T)=\theta Q(t), \quad \theta:=\frac{c^{+}+c^{-}-\sigma c^{+} c^{-}}{c^{+}+c^{-}+\sigma c^{+} c^{-}} \in(-1,1) .
$$

It then follows that for each integer $n$,

$$
Q(t+n T)=\theta^{n} Q(t)
$$

Consequently, $Q(t)$ exponentially approaches zero as $t \rightarrow \infty$. From this, we conclude from $\dot{s}_{1}(t)=\frac{1}{\alpha^{-}-\alpha^{+}} Q(t)$ that $s_{1}(t)$ approaches a limit exponentially fast.

A further special noteworthy case is

$$
\sigma c^{+} c^{-}=c^{+}+c^{-} \Longleftrightarrow \sigma=1 / c^{+}+1 / c^{-} \Longleftrightarrow \theta=0 .
$$

In this case, $Q \equiv 0 \forall t>T$; namely, the interface reaches its final destination at time $T$ and does not move thereafter. (This does not mean that the system enters a steady state at time $T$ since $\left(u_{1}^{ \pm}, v_{1}^{ \pm}\right)$may not be zero.)

Also, one sees that when $\theta<0$, i.e., $\sigma>1 / c^{+}+1 / c^{-}, Q(t)$, and also $\dot{s}_{1}$, changes sign infinitely many times; that is to say, the interface oscillatorily reaches its final destination.

On the other hand, if $\sigma<1 / c^{-}+1 / c^{+}$, then $\theta>0$, and for initial data satisfying $Q(t) \geq 0$ in $(0, T)$ (which is equivalent to $\left[u_{0}\left(s_{0}+c^{+} t\right)+u_{0}\left(s_{0}+c^{-} t\right)+v_{0}\left(s_{0}+\right.\right.$ $\left.\left.\left.c^{+} t\right) / c^{+}+v_{0}\left(s_{0}-c^{-} t\right) / c^{-}\right] \geq 0 \forall t \in[0, T]\right), Q(t) \geq 0$ and $\dot{s}_{1} \leq 0 \forall t>0$; namely, the interface approaches its final destination monotonically.

We remark that condition (3.15) depends only on the state function $p=p(u)$.

3.3. A separation of variable approach. In order to find more detailed behavior of solutions of (P1), we decompose solutions into their Fourier modes. Hence, ignoring initial conditions, we seek special solutions of the form

$$
u_{1}=e^{\lambda t} \phi(x, \lambda), \quad v_{1}=e^{\lambda t} \psi(x, \lambda), \quad \dot{s}_{1 t}=\frac{\sigma}{\alpha^{-}-\alpha^{+}}\left(c^{+}\right)^{2} \phi\left(s_{0}+, \lambda\right) e^{\lambda t} .
$$


Substituting this form into the system, we see that it is a special solution if and only if $(\phi, \psi, \lambda)$ solves the eigenvalue problem

$$
\left\{\begin{array}{l}
\psi^{\prime}=\lambda \phi, \quad x \in \Omega:=(-a, a) \backslash\left\{s_{0}\right\}, \\
c^{2} \phi^{\prime}=\lambda \psi, \quad x \in \Omega \\
\psi(-a)=\psi(a)=0, \\
\psi\left(s_{0}+\right)-\psi\left(s_{0}-\right)=\sigma\left(c^{+}\right)^{2} \phi\left(s_{0}+\right)=\sigma\left(c^{-}\right)^{2} \phi\left(s_{0}-\right) .
\end{array}\right.
$$

THEOREM 3.3. Let $T^{ \pm}=\left(a \mp s_{0}\right) / c^{ \pm}$be the time needed for the sound wave to travel from the interface $x=s_{0}$ to the boundary $x= \pm a$ in the $\alpha^{ \pm}$phase. Consider the eigenvalue problem (EVP) for $(\lambda, \phi, \psi)$. The following holds:

(1) All eigenvalues have nonpositive real part;

(2) There is a real eigenvalue if and only if

$$
\sigma<1 / c^{+}+1 / c^{-} .
$$

If the above holds, then there is only one real eigenvalue, and it is strictly negative.

(3) Assume that $T^{+} / T^{-}=p / q$ is a rational number with indivisible odd integers $p$ and $q$. Then there are infinitely many pure imaginary eigenvalues, and the corresponding eigenfunctions $(\phi, \psi)$ satisfy $c^{2} \phi, \psi \in C^{1}([-a, a])$, and $\phi\left(s_{0}+\right)=\phi\left(s_{0}-\right)=0$.

(4) Assume either that $T_{+} / T^{-}$is an irrational number or $T^{+} / T^{-}=p / q$ with indivisible integers $p$ and $q$, one of them being even. Then all eigenvalues have negative real part, and every eigenfunction $(\phi, \psi)$ has the property that $\phi\left(s_{0} \pm\right) \neq 0$.

We remark that for any real eigenvalue, the corresponding $\dot{s}_{1}$ does not change sign, so as $t \rightarrow \infty, s_{1}(t)$ tends to its limit monotonically.

Proof. One notices that $\lambda=0$ is not an eigenvalue.

The general solutions to the ODEs in (EVP) with the boundary conditions $\psi( \pm a)$ $=0$ are

$$
\left\{\begin{array}{l}
\psi(x)=c^{ \pm} A^{ \pm} \sinh \left[\lambda(x \mp a) / c^{ \pm}\right], \\
\phi(x)=A^{ \pm} \cosh \left[\lambda(x \mp a) / c^{ \pm}\right],
\end{array} \quad \pm\left(x-s_{0}\right) \in\left(0, a \mp s_{0}\right),\right.
$$

where $A^{ \pm}$are arbitrary constants. For $(\phi, \psi, \lambda)$ to be a nontrivial solution to (EVP), we need only $\left|A^{+}\right|+\left|A^{-}\right|>0$ and

$$
\begin{aligned}
\sigma A^{+}\left(c^{+}\right)^{2} \cosh \left[\lambda T^{+}\right] & =\sigma A^{-}\left(c^{+}\right)^{2} \cosh \left[\lambda T^{-}\right] \\
& =-c^{+} A^{+} \sinh \left[\lambda T^{+}\right]-c^{-} A^{-} \sinh \left[\lambda T^{-}\right] .
\end{aligned}
$$
$\neq 0$.

We consider two cases: (i) $\cosh \left[\lambda T^{+}\right] \cosh \left[\lambda T^{-}\right]=0$, (ii) $\cosh \left[\lambda T^{-}\right] \cosh \left[\lambda T^{+}\right]$

First we consider case (i). Then either $\cosh \left[\lambda T^{+}\right]=0$ or $\cosh \left[\lambda T^{-}\right]=0$. Suppose $\cosh \left[\lambda T^{+}\right]=0$. Then we cannot have $A^{-}=0$ since it would imply from (3.16) that $A^{+}=0$. Hence, $\cosh \left[\lambda T^{-}\right]=0$. Similarly, if $\cosh \left[\lambda T^{-}\right]=0$, we can derive $\cosh \left[\lambda T^{+}\right]=0$. Thus,

$$
\cosh \left[\lambda T^{+}\right]=\cosh \left[\lambda T^{-}\right]=0 .
$$

Now suppose there is a $\lambda$ satisfying the above condition. Then $\left|\sinh \left[\lambda T^{+}\right]\right|=\left|\sinh \left[\lambda T^{-}\right]\right|$ $=1$ and we can simply take $A^{-}=1$ and $A^{+}=-c^{-} \sinh \left[\lambda T^{-}\right] /\left(c^{+} \sinh \left[\lambda T^{+}\right]\right)$to obtain a solution to (EVP); in other words, (3.17) is a sufficient condition for $\lambda$ to be 
an eigenvalue. As we shall see later, case (ii) will not produce any pure imaginary eigenvalues, and we conclude that $\lambda$ is a pure imaginary eigenvalue if and only if (3.17) holds. If (3.17) holds, then the corresponding eigenfunction $(\psi, \phi)$ has the property that $\phi\left(s_{0} \pm\right)=0$ and both $c^{2} \phi$ and $\psi$ are in $C^{1}([-a, a])$.

Now we solve (3.17). Since the only solution to $\cosh [z]=0$ is $z=\mathrm{i}[\pi / 2+\mathrm{k} \pi]$ for all integers $k$, we must have, for some integers $k$ and $l, \lambda T^{+}=\mathrm{i}(\pi / 2+k \pi) / T^{-}$and $\lambda T^{-}=\mathrm{i}(\pi / 2+l \pi)$. This implies that $T^{+} / T^{-}=(1+2 k) /(1+2 l)=p / q$, where $p$ and $q$ are odd integers indivisible to each other.

Now assume that $T^{+} / T^{-}=p / q$ is a rational number where $p$ and $q$ are odd integers not divisible to each other. Then it is easy to check that all solutions of (3.17) are given by

$$
\lambda=\lambda_{n}=\frac{n p \pi}{2 T^{+}} \mathrm{i}=\frac{n q \pi}{2 T^{-}} \mathrm{i}, \quad n= \pm 1, \pm 3, \pm 5, \ldots
$$

This proves assertion (3).

Next we consider case (ii): $\cosh \left[\lambda T^{+}\right] \cosh \left[\lambda T^{-}\right] \neq 0$. Then we must have $A^{\mp} \neq 0$. Hence, we can without loss of generality take $A^{ \pm}=\left(c^{ \pm}\right)^{-2} \cosh \left[\lambda T^{ \pm}\right]$and conclude that $\lambda$ is an eigenvalue if and only if

$$
\sigma+\frac{1}{C^{+}} \tanh \left[\lambda T^{+}\right]+\frac{1}{C^{-}} \tanh \left[\lambda T^{-}\right]=0 .
$$

From this equation, we immediately obtain the second assertion (2) of the theorem.

Write $\lambda=\mu+\mathrm{i} \omega$ where $\mu$ and $\omega$ are real. Then (3.18) is equivalent to find $(\mu, \omega)$ such that

$$
\left\{\begin{array}{l}
\frac{\sin \left(2 \omega T^{-}\right)}{c^{-}\left[\cos ^{2}\left(\omega T^{-}\right)+\sinh ^{2}\left(\mu T^{-}\right)\right]}+\frac{\sin \left(2 \omega T^{+}\right)}{c^{+}\left[\cos ^{2}\left(\omega T^{+}\right)+\sinh ^{2}\left(\mu T^{+}\right)\right]}=0, \\
\sigma+\frac{\sinh \left(2 \mu T^{+}\right)}{2 c^{+}\left[\cos ^{2}\left(\omega T^{+}\right)+\sinh ^{2}\left(\mu T^{+}\right)\right]}+\frac{\sinh \left(2 \mu T^{-}\right)}{2 c^{-}\left[\cos ^{2}\left(\omega T^{-}\right)+\sinh ^{2}\left(\mu T^{-}\right)\right]}=0 .
\end{array}\right.
$$

From this, the remaining assertions of the theorem follow. This completes the proof of the theorem.

Once the eigenvalue problem is solved, the solution to $(\mathrm{P} 1)$ can be written as a Fourier series. We omit the details.

We remark that when $T^{+} / T^{-}$is irrational, there is no negative upper bound for the real part of all eigenvalues; namely, the dissipation is there, but it can be arbitrarily small. Nonetheless, we can still conclude that the functions $(\mathbf{u}, \hat{v})$ in the proof of Theorem 3.1 are actually identically zero, i.e., $\mathbf{u}=\hat{v} \equiv 0$, so that all solutions approach a trivial limit as $t \rightarrow \infty$. Therefore, we have the following result.

TheOrem 3.4. (1) Assume that either $T^{+} / T^{-}$is irrational or $T^{+} / T^{-}=p / q$ with indivisible integers $p$ and $q$, one of them being even. Then all solutions to problem (P1) satisfy

$$
\lim _{t \rightarrow \infty} u_{1}^{ \pm}=\lim _{t \rightarrow \infty} v_{1}^{ \pm}=0, \quad \lim _{t \rightarrow \infty} s_{1}(t)=s_{0}+\frac{1}{\alpha^{+}-\alpha^{-}} \int_{-a}^{a} u(\cdot, 0) .
$$

(2) Assume that $T^{+} / T^{-}=p / q$ for some indivisible odd integers $p$ and $q$. Then the boundary value problem wave equations in (P1) admit infinitely many linearly independent time periodic solutions $\left(u_{1}, v_{1}\right)$ satisfying $c^{2}(\cdot) u_{1}(\cdot, t), v_{1}(\cdot, t) \in C^{1}([-a, a])$ and $u_{1}\left(s_{0} \pm, t\right)=0 \forall t \in R$. 
4. Numerical results. We solve (1.1) with a second order finite difference method. $p(u)$ is chosen to be the van der Waals equation of state

$$
p(u)=\frac{1}{u-0.25}-\frac{0.9}{u^{2}} .
$$

The Maxwell states are $\alpha^{+}=1.405065$ and $\alpha^{-}=0.494273$. The corresponding wave speeds are $c^{+}=0.317204, c^{-}=1.36113$. We start with initial condition

$$
\left\{\begin{array}{l}
u(x, 0)=\frac{\alpha^{+} e^{4 x}+\alpha^{-} e^{-4 x}}{e^{4 x}+e^{-4 x}}, \\
v(x, 0)=0.01 e^{-4 x^{2}}
\end{array}\right.
$$

where $u(x, 0)$ is a small perturbation around a phase jump connecting $\alpha^{+}$and $\alpha^{-}$. When $x$ is positive and large, $u(x, 0)$ is close to $\alpha^{+}$. When $x$ is negative and large, $u(x, 0)$ is close to $\alpha^{-}$. Figures 1 and 2 show that the perturbation in the region $\Omega_{+}$ is traveling with wave speed $c^{+}$and the perturbation in the region $\Omega_{-}$is traveling with wave speed $c^{-}$(which is much faster than $c^{+}$). When a perturbation reaches the boundary, it is reflected back and travels in the opposite direction. The interface also moves back and forth slightly and eventually approaches a steady state.
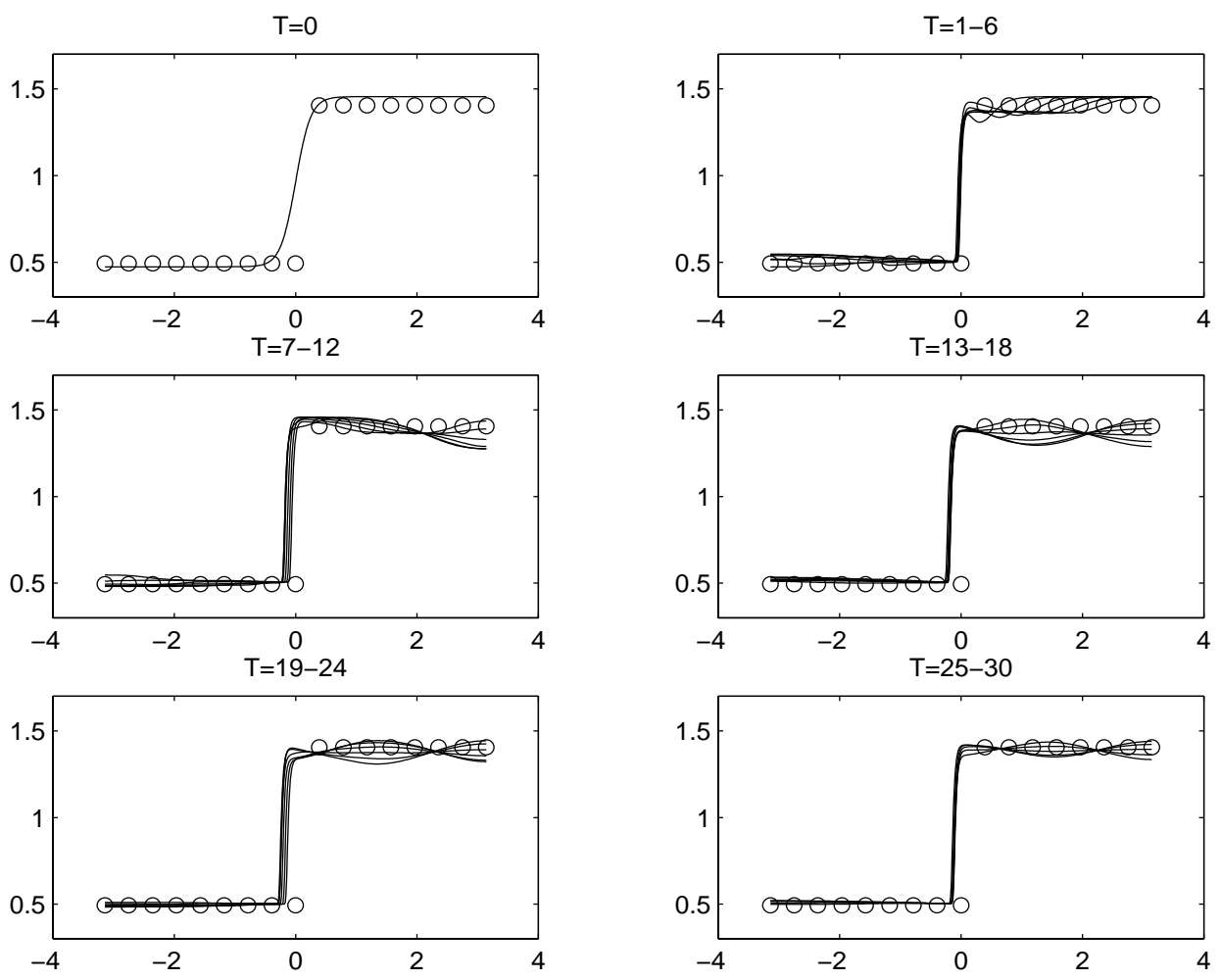

FIG. 1. Evolution of $u(x, t)$. Circles represent values a and $b$. 
$\mathrm{T}=30-35$
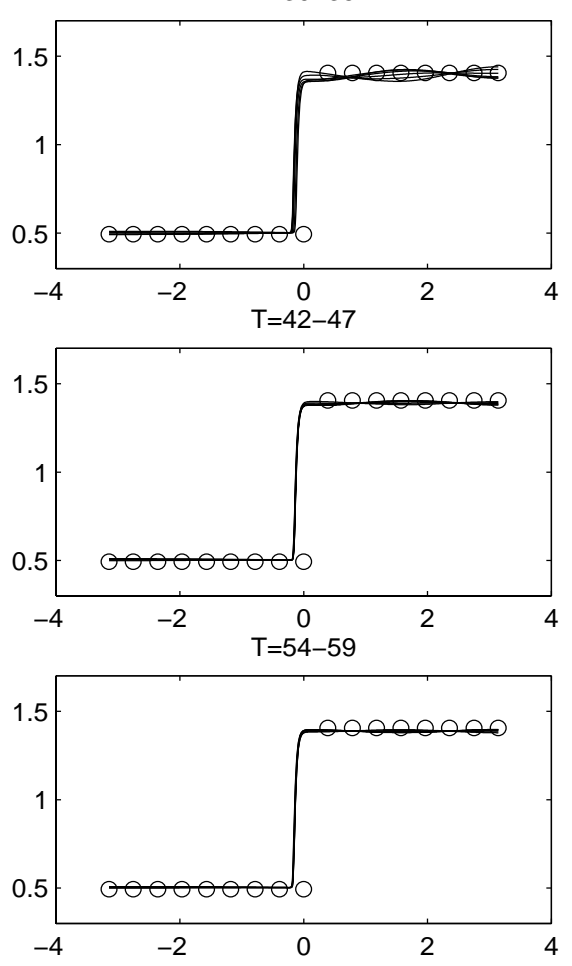

$\mathrm{T}=36-41$
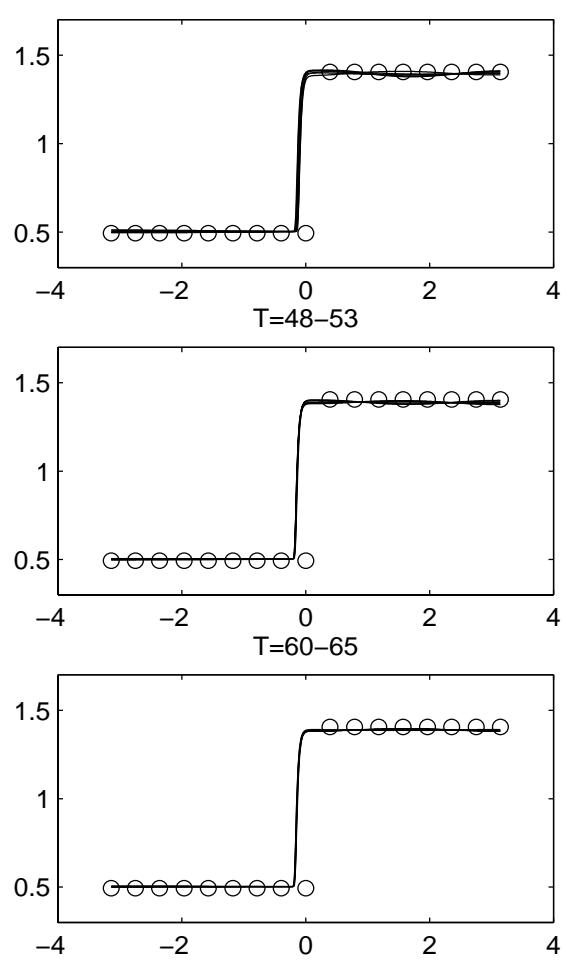

FIG. 2. Continuation of Figure 1.

\section{REFERENCES}

[1] N. D. Alikakos, P. W. Bates, and Xinfu Chen, Convergence of the Cahn-Hilliard equation to the Hele-Shaw model, Arch. Rational Mech. Anal., 128 (1994), pp. 165-205.

[2] G. Caginalp And X. Chen, Convergence of the phase field model in its sharp interface limits, European J. Appl. Math., 9 (1998), pp. 417-445.

[3] J. Carr, M. E. Gurtin, and M. Slemrod, Structured phase transitions on a finite interval, Arch. Rational Mech. Anal., 86 (1984), pp. 317-351.

[4] X. Chen, Rigorous verification of formal asymptotic expansions, in Proceedings of the International Conference on Asymptotic in Nonlinear Diffusive Systems, Tohoku Math. Publ., (1998), pp. 9-33.

[5] T.-P. LiU, Nonlinear stability of shock waves for viscous conservation laws, Mem. Amer. Math. Soc., 56 (1985).

[6] H. T. FAN, A limiting viscosity approach on the dynamics of phase transitions in van der Waals fluids, J. Differential Equations, 103 (1993), pp. 179-204.

[7] P. De Mottoniand and M. Schatzman, Geometrical evolution of developed interfaces, Trans. Amer. Math. Soc., 347 (1995), pp. 1522-1589.

[8] M. SHEARER, Dynamic phase transitions in a van der Waals gas, Quart. Appl. Math., 46 (1988), pp. 631-636.

[9] K. Zumbrun, Dynamical Stability of Phase Transitions in the p-System with ViscosityCapillarity, preprint.

[10] R. Hagan and M. Slemrod, The viscosity-capillarity criterion for shocks and phase transitions, Arch. Rational Mech. Anal., 83 (1984), pp. 333-361.

[11] D.-Y. Hsien AND X.-P. WANG, Phase transition in van der Waals fluid, SIAM J. Appl. Math., 57 (1997), pp. 871-892.

[12] P. Fife And X. P. Wang, Periodic structures in a van der Waals system, Proc. Roy. Soc. Edinburgh Sect. A, 128 (1998), pp. 235-250. 
[13] C. He And X. P. Wang, Symmetric Solutions for a Two Dimensional van der Waals System, preprint.

[14] M. Slemrod, Admissibility criteria for propagating phase boundaries in a van der Waals fluid, Arch. Rational Mech. Anal., 81 (1983), pp. 301-315.

[15] A. Szepessy And Z. Xin, Nonlinear stability of viscous shock waves, Arch. Rational Mech. Anal., 122 (1993), pp. 53-103

[16] L. ModicA, The gradient theory of phase transitions and minimal interface criterion, Arch. Rational Mech. Anal., 98 (1987), pp. 123-142.

[17] R. V. Kohn And P. SternberG, Local minimizers and singular perturbations, Proc. Roy. Soc. Edinburgh Sect. A, 111 (1989), pp. 69-84. 